\title{
Eine Monografie über die Struktur der Ortsnamen der Saamen
}

TAARna VALtonen: Mielen laaksotMielen vuomieh - Miela vuomit Mielâ vyemeh - Miõl vue'm. Neljän saamen kielen paikannimien rakenne, sanasto ja rinnakkaisnimet vähemmistö-enemmistösuhteiden kuvastajina. [Struktur, Lexik und Parallelbezeichnungen der Ortsnamen in vier saamischen Sprachen als Indikatoren der Beziehungen zwischen Minderheit und Mehrheit.] Suomalais-Ugrilaisen Seuran Toimituksia 271. Helsinki 2014. $452+88$ S.

\section{Allgemeines}

Die Dissertation von Taarna Valtonen ist die erste umfangreiche vergleichende Untersuchung über die Ortsnamen in verschiedenen saamischen Sprachen.

Untersuchungsgegenstand sind die südsaamischen, nordsaamischen, inarisaamischen und skoltsaamischen Ortsnamen, die im Licht der Namen aus vier verschiedenen Beispielregionen betrachtet werden. $\mathrm{Zu}$ diesen Namen werden eine Strukturanalyse, eine etymologische Analyse der in den $\mathrm{Ne}$ men verwendeten Lexik sowie bei
Namen, die in mehreren Sprachen begegnen, eine Analyse der $\mathrm{Na}$ menspaare vorgelegt. Die Ergebnisse werden im Kontext der Ethnografie und der Sprachkontaktforschung interpretiert. Der Band enthält ferner eine recht umfangreiche ethnografische Darstellung der untersuchten Gemeinschaften und ihres Wandels.

Valtonens Untersuchung ist weitaus umfangreicher als durchschnittliche Dissertationen und stützt sich auf größere Korpora. Diese hätten durchaus genug Stoff für mehrere Dissertationen geboten, die wahrscheinlich ebenfalls angenommen worden wären. Die Untersuchung ist durchgängig von hoher Qualität, der sprachliche Stil und der Aufbau sind ausgefeilt, und die Gesamtheit vermittelt einen durchdachten und ausgearbeiteten Eindruck. Die Ergebnisse der Untersuchung sind in vielen Teilen bedeutsamer als in durchschnittlichen Dissertationen und dürfen als dauerhaft gelten.

Die Arbeit knüpft an mindestens drei wissenschaftsgeschichtliche Traditionen an, nämlich an die ethnografische Forschung über die Saamen, an die Tradition der 
historisch-vergleichenden Untersuchung der saamischen und darüber hinaus der uralischen Sprachen sowie drittens an die finnische syntaktisch-semantische Tradition der Ortsnamenforschung. In allen diesen Bereichen liefert Valtonen neue Kontributionen. Es sei ausdrücklich erwähnt, dass es sich um die erste Darstellung der strukturellen Eigenschaften der Ortsnamen in vier verschiedenen Sprachen handelt, was als einzigartiges Verdienst gelten darf.

In den Bereich der Grundlagenforschung gehört die Darlegung der strukturellen Eigenschaften, der Lexik und der zweisprachigen Namenspaare der saamischen Ortsnamen. Sie bietet für die künftige Forschung wichtige, grundlegende Informationen über die saamischen Ortsnamen. Aus der Sicht der Sprachkontakte handelt es sich um eine Untersuchung zur Siedlungs- und Kulturgeschichte, die nicht nur mit der historischen Sprachwissenschaft, sondern auch mit den Forschungstraditionen im Bereich der Vorgeschichte verknüpft ist. Die Verfasserin ist mit Theorie und Praxis der Ethnografie und der Namensforschung gründlich vertraut. Im Bereich der historischen Uralistik ist die Herangehensweise referierender und stärker an die einschlägige Literatur und die mündlichen Hinweise des Betreuers gebunden.

Die Verfasserin hat sich entschieden, ihre Arbeit auf Finnisch $\mathrm{zu}$ schreiben, wodurch sie dem größten Teil der potentiell interessierten Leserschaft zugänglich ist. Diese Wahl ist in der heutigen Situation der humanistischen Forschung besonders lobenswert, denn die vielsprachige europäische ethnografische Forschungstradition droht $\mathrm{zu}$ verkümmern und sich an angelsächsische Muster anzupassen. Der ethnografischen Erforschung der Minderheitsvölker Eurasiens hat die englischsprachige Forschungstradition jedoch nicht allzu viel zu bieten. In dieser Hinsicht ist die Aufrechterhaltung der finnischsprachigen Forschungstradition bei Untersuchungen über die Völker des Nordens ein wissenschafts- und kulturpolitisch bedeutsamer Akt. Es sei dennoch angemerkt, dass Valtonen, da es sich um eine verdienstvolle Untersuchung handelt, zumindest ihre wichtigsten Ergebnisse auch in anderen Sprachen publizieren sollte, vor allem in den saamischen und skandinavischen Sprachen sowie auf Englisch. 


\section{Untersuchungsgegenstand und -material}

Die untersuchten Gebiete sind das zum Verbreitungsgebiet des Südsaamischen gehörende Gebiet von Ruvhten sijte in Härjedalen in Schweden, das zum Verbreitungsgebiet des Nordsaamischen gehörende Gebiet des Dorfes Dálvadaš in Utsjoki, das zum Verbreitungsgebiet des Inarisaamischen gehörende Gebiet Čovčjävri-Kosseennâm im nördlichen Teil der Gemeinde Inari und das zum Verbreitungsgebiet des Skoltsaamischen gehörende Gebiet der Familie Sverloff in Suonikylä im ehemaligen Petsamo, das heute zu Russland gehört.

Die Gebiete wurden so ausgewählt, dass sie unterschiedliche saamische Sprachen und Gemeinschaften repräsentieren. Um ein Gesamtbild der Ortsnamen in den saamischen Sprachen zu erhalten, wäre es wünschenswert gewesen, auch die saamischen Sprachen auf der Halbinsel Kola einzubeziehen, doch geeignetes Material dürfte nicht leicht zu finden sein. Im Hinblick auf die Betrachtung der Gewerbe und der unterschiedlichen kulturellen Kontexte wiederum hätten die seesaamischen Ortsnamen sicherlich Material geboten, das von den in der Untersuchung verwendeten Korpora merklich abweicht. Doch schon das jetzige
Material ist in der Geschichte der Lappologie von einzigartigem Umfang. In der Regel behandeln Dissertationen nur eine der saamischen Sprachen.

Das Material aus den verschiedenen Gebieten ist vom Umfang und auch vom Typ her sehr unterschiedlich. Die Zahl der südsaamischen Namen beträgt 168 , die der nordsaamischen 222, der inarisaamischen 561 und der skoltsaamischen 655. Das Untersuchungsmaterial wird im Anhang präsentiert. Bei dem Material aus Ruvhten sijte handelt es sich um das Namenmaterial eines gesamten historischen Saamendorfes (siida), während die anderen Korpora jeweils nur ein von einigen Familien bewohntes Gebiet abdecken. Dennoch ist das Material aus Ruvhten sijte vom Umfang her das kleinste und enthält die wenigsten Mikronamen. Es ist daher möglich, dass die in der Untersuchung aufgezeigten Unterschiede der Benennungspraxis im Südsaamischen und den anderen Sprachen teilweise auch darauf zurückgehen. Die Verfasserin ist sich dieses Problems bewusst und kommt immer wieder auf den speziellen Charakter des Materials von Ruhvten sijte zurück.

Ein zweites mit dem Material verknüpftes Problem ist, dass bei dem Material aus Dálvadas und Čovčjävri-Kosseennaam eine 
erhebliche Variation bei der Verwendung bestimmter Namen aufgezeigt werden konnte, während entsprechende Angaben für Ruvhten sijte und Suonikylä nicht verfügbar sind. Daher ist das von der Verfasserin verwendete Material nicht völlig vergleichbar. Es dürfte einleuchtend sein, dass z. B. die meisten einteiligen, örtlich verwendeten Namensvarianten (elliptische Namen) dort registriert werden können, wo das größte die Variation veranschaulichende Material vorliegt.

Obwohl Valtonen in den Verbreitungsgebieten der verschiedenen saamischen Sprachen auch Feldforschung betrieben hat, präsentiert sie kaum präzisierendes und ergänzendes Material aus ihrer eigenen Sammlung. Besonders für das Südsaamische wäre eine neuere Sammlung notwendig gewesen, denn viele Aspekte des Materials können nicht erschöpfend erklärt werden. Andererseits tritt die Kenntnis des saamischen Kulturkontextes, die sich die Verfasserin durch die Feldforschung erworben hat, an vielen Stellen hervor und kompensiert dieses Manko.

$\mathrm{Zu}$ einem gewissen Grad problematisch ist das Bestreben der Verfasserin, die Untersuchung auf die zu Beginn des 20. Jahrhunderts gebräuchlichen „traditionellen“ Namen zu beschränken (S. 31).
Man weiß nämlich, dass die kulturelle Situation in der ersten Hälfte des 20. Jahrhunderts in den saamischen Gebieten in vielen Fällen ebenfalls erst kürzlich entstanden war. Beispielsweise hatte sich in Inari die überwiegend auf Binnenseefischfang, Rentier- und Viehzucht sowie dem Wechsel zwischen Sommer- und Winterwohnstätten beruhende Kulturform erst im Lauf des 19. Jahrhunderts etabliert, als Großrentierzüchter aus Norwegen eintrafen, das Waldren verschwand und neue Erwerbszweige die Waldrenjagd ersetzten. Im 18. Jahrhundert hatte der Umzug der Gemeinschaft aus Zelten in feste Häuser begonnen. Gleichzeitig hatte sich das Zentrum der Gemeinschaft aus den Winterdörfern am Fluss Nukkumajoki nach Pielpajärvi verschoben; die Bevölkerung war zum Christentum übergetreten und hatte ihre alten religiösen Traditionen aufgegeben. Die vorhergehenden Jahrhunderte wiederum hatten zunächst die konkurrierende Besteuerung durch drei Staaten und die Integrierung in die europäischen Handelsverkehrsnetze gebracht. Entsprechende Veränderungen durchlief zumindest auch das südsaamische Gebiet. Die saamische Ethnie befand sich also in ständigem Wandel, und ihre Ortsnamen spiegeln die kulturelle Situation 
verschiedener Epochen wider, die sich nicht einfach in eine moderne und eine vormoderne Zeit gliedern lässt.

Die Vorstellung von recht stabilen saamischen Gemeinschaften kommt auch darin zum Ausdruck, dass die Verfasserin zwar verdienstvoll die Kulturkontakte schildert, die sich in den Namenssystemen der verschiedenen Gebiete widerspiegeln, aber kaum auf die Möglichkeit hinweist, dass es zwischen den saamischen Gruppen oder zwischen den Saamen und den Vertretern der dominierenden Kultur ethnische Assimilationsprozesse gegeben haben könnte. Z. B. deutet das auf strenger Teilung der Erwerbsarten und einem geteilten Namenssystem (in den Waldgebieten) beruhende System von Ruvhten sijte darauf hin, dass die Menschen, wenn sie die schwedisch-saamische ethnische Grenze überschritten, auch die Sprache und die ethnische Identität wechselten. Die von der Verfasserin beobachtete Fülle von Bestimmungsgliedern skandinavischer Herkunft ist möglicherweise so zu interpretieren, dass die skandinavische Besiedlung zumindest in Teilen des Gebiets älter ist als die saamische und dass die heutige Sprachgrenze entstand, als sich die Grenze zwischen den Erwerbszweigen festigte. Die Frage ist allerdings äußerst kompliziert, wie die Verfasserin zu Recht feststellt.

Die inari-nordsaamischen $\mathrm{Na}$ menpaare von Dálvadas und Čovčjävri-Kosseennâm wiederum sind so geartet, dass die Frage, in welcher der beiden Sprachen sie zuerst entstanden sind, nicht unbedingt begründet ist. Die Namen können auch die Namentradition aus der Zeit vor der Entstehung der heutigen saamischen Sprachen widerspiegeln oder bei den Kontakten von Sprechern zweier verschiedener Formen des Saamischen entstanden sein. Obwohl die lappologische Forschungstradition die Inarisaamen und die Saamen von Dálvadas verschiedenen ethnischen Gruppen zuord net, fassten die in der Gegend lebenden Menschen in der Vergangenheit die Grenzen zwischen den Ethnizitäten wahrscheinlich pragmatischer auf. Gewisse Umstände, u. a. areallinguistische Schlussfolgerungen und einige Ortsnamen, deuten auch darauf hin, dass im Flusstal des Teno früher eine Sprachform gesprochen wurde, die größere Ähnlichkeit mit dem Inarisaamischen (oder Ostsaamischen) aufwies. Daher hätte ein etwas dynamischerer Ausgangspunkt für das Verständnis der Geschichte der zu untersuchenden Gemeinschaften vielleicht an manchen Stellen die Perspektive auf die Zeit vor der 
Entstehung der heutigen Gruppenidentitäten erweitert.

Ein entsprechender, leicht essentialistischer Ausgangspunkt tritt auch dann zu Tage, wenn die Verfasserin über die Sprache schreibt. Sie spricht z. B. von Faktoren, die eine Gemeinschaft und eine Sprache „schützen“ oder „verderben“, gemäß der puristischen Tradition der Sprachpflege. Das kann man natürlich tun, doch beim heutigen Stand der humanistischen Forschung wäre es vielleicht angebracht, auch auf das dekonstruktivistische Paradigma (z. B. Jan Blommaert, Ben Rampton) zu verweisen, in dem die kontinuierliche Konstruktion ethnischer und gemeinschaftlicher Identitäten, die Offenheit der Sprachen als Systeme und die Position von Sprachformen mit zahlreichen Entlehnungen oder Codewechseln als vollwertige und echte Sprachen hervorgehoben werden.

\section{Methoden und Theorien}

Indigene Völker betreffende Forschungskonstellationen, in denen versucht wird, die traditionellen Auffassungen von ethnischen Gemeinschaften, von ihren Sprachformen oder ihrem kulturellen Wissen zu dekonstruieren, haben keinen nennenswerten Einfluss auf die vorliegende Untersuchung gehabt. Das hat den Vorteil, dass die Untersuchung nahtlos an die Tradition anknüpft, theoretisch auf solider Basis steht und neue, dauerhafte Resultate im Bereich der Basisforschung erbringt. Andererseits erscheint die als theoretische Inspiration dienende Gliederung von Minderheiten und Mehrheiten in sichere und unsichere (Liebkind) nicht immer als ausreichendes Instrument für die Analyse der verschiedenen Gebiete. Die Verfasserin hätte auch auf die Theoretiker des sozialen Konstruktivismus zurückgreifen können, die den kontinuierlichen Wandel von Gemeinschaftsidentitäten betonen.

Valtonen bezeichnet ihre Methodik als kulturelle Onomastik. Es handelt sich offenbar um einen von ihr selbst geschaffenen Terminus. Im Theoriekapitel (z. B. S. 55-62) beschreibt die Verfasserin das Verhältnis zwischen den Ortsnamen und dem kulturellen Wissen in vielerlei Hinsicht verdienstvoll und aus der Sicht der finnischen Onomastik sogar bahnbrechend. Dennoch wird nicht völlig klar, in welcher Beziehung die kulturelle Onomastik zur lexikalischen, syntaktisch-semantischen und etymologischen Analyse steht, abgesehen davon, dass die Ergebnisse in ihrem ethnografischen und historischen Kontext interpretiert werden. 
Die Verfasserin ist sich des Problems bewusst, dass die Methoden der finnischen Onomastik nicht optimal geeignet sind, um das kulturelle Weltbild, das die saamischen Ortsnamen widerspiegeln, oder die Verbindungen zwischen der Kultur und der Struktur der Namen zu analysieren. Sie kennt die Geschichte und die ethnografischen Besonderheiten der von ihr untersuchten Gemeinschaften. Die vorgelegte Analyse des Namenmaterials ist dennoch recht traditionell und richtet sich auf die Herkunft und die Verwendung der in den Namen begegnenden Wörter. Valtonen analysiert die Struktur der Namen und den darin auftretenden Wortschatz äußerst sorgfältig, erörtert aber nicht die Benennungsmotivationen aus der Perspektive der Modellmethode. Da die Verfasserin weniger die Motivationen der Benennung erörter, sonder sich auf den in den Namen vorkommenden Wortschatz konzentriert, werden einige Namen möglicherweise falsch erklärt. Beispielsweise gibt es der Verfasserin zufolge im saamischen Namenschatz kaum Namen, die mit Grenzen zu tun haben. Es scheint jedoch denkbar, dass solche unter denjenigen Namen zu finden sind, in denen ein Wortschatz vorkommt, der heute nicht auf Grenzen verweist. U. a. haben die Namen mit dem Element siida am Inarisee (z. B. die Namen kleiner Klippen) natürlich nichts mit den Winterdörfern zu tun, sondern scheinen der von Voitto Viinanen (2006) rekonstruierten älteren siita-Grenze $\mathrm{zu}$ folgen. Entsprechend sind viele Namen, die einen Personennamen enthalten, möglicherweise auch als Angabe eines Besitzverhältnisses und somit als eine Art Grenznamen $\mathrm{zu}$ interpretieren.

Auch wenn die Benennungsmodelle kaum im Fokus einer verschiedene Gebiete vergleichenden Untersuchung stehen können, wäre es dennoch möglich gewesen, die Unterschiede der Benennungsmotivationen in der saamischen Kultur und den sie umgebenden Kulturen sowie die größeren Schemata zu erörtern, die im jeweiligen Kulturkontext die Benennung lenken. Die Verfasserin hat bei der Theoriebildung hauptsächlich skandinavische und angelsächsische Quellen verwendet, die gerade im Bereich der Namenforschung im Allgemeinen an die Richtungen der strukturalistischen Linguistik gebunden sind. Auch in Finnland wurde jedoch eine kognitive Onomastik entwickelt, die danach strebt, über die Klassifizierung der Motivationen einzelner Namen hinaus auf die Ebene der Beschreibung der Schemata zu gelangen, die das $\mathrm{Na}$ menssystem steuern (Leino 2007), und es wäre interessant gewesen, zu 
erfahren, wie die Verfasserin die in ihrer Untersuchung zentralen, mit der saamischen Kultur verknüpften Befunde in solche Schemata einordnet. Ähnliche Ziele verfolgten in der älteren Fennistik die onomasiologische Tradition, die unterschiedliche Motivationen der Benennung im appellativen Wortschatz untersuchte (u. a. die Arbeiten von R.E. Nirvi und Mauno Koski) sowie die russische ethnolinguistische Tradition (z. B. Berezovič 2012).

Diese Bemerkungen mögen wie Kritik wirken, doch es handelt sich eher um den Versuch, das in der Untersuchung Dargelegte zu kontextualisieren. Valtonen konzentriert sich in ihrer Untersuchung stark auf das Material und vermeidet theoretische Spekulationen. Das ist insofern lobenswert, als die Resultate auf sicherem Fundament stehen, andererseits gibt das Material stellenweise nicht so viel her, wie es bei einer ehrgeizigeren theoretischen Analyse möglich gewesen wäre.

Der finnougristische Kontext beschränkt sich auf das Ostseefinnische und die saamischen Sprachen. Möglicherweise hätte die russische Forschung zu einem gewissen Grad einen nützlichen Kontext für die Analyse der saamischen Namen liefern können. Z. B. scheint die wichtige und umfangreiche Untersuchung von Tatjana Dmitrieva über die Ortsnamen der
Chanten (Dmitrieva 2005) zusammen mit Valtonens Untersuchung zu zeigen, dass die Ortsnamen der Jäger-Sammler und Hirten sich von den syntaktisch-semantischen Typen her häufig von den Namen der Bodenbauer unterscheiden. Die Namen haben im Durchschnitt mehr Bestandteile und die Namenskluster sind größer. Das dürfte damit zusammenhängen, dass das durchschnittliche Namensrevier des Jäger-Sammlers und des Hirten größer ist als das des Bodenbauers.

\section{Analyse und Schlussfolgerungen}

Die in der Dissertation vorgelegte empirische Analyse des Materials ist durchgängig zuverlässig und sorgfältig. Neue Untersuchungsergebnisse von dauerhafter Bedeutung sind u. a. die Darstellung der lautlichen Reduktionserscheinungen im saamischen Namensgut, die Zählung der im Namensgut vorkommenden Namensteile und die Erläuterung des in den $\mathrm{Na}$ men am häufigsten begegnenden Wortschatzes sowie der Typen von Namenspaaren verschiedener Sprachen. Die Grundzüge der Ortsnamen der saamischen Sprachen sind nun mindestens so gut dargestellt wie die einiger ostseefinnischer Sprachen (Kiviniemi 1990 [Finnisch], Mullonen 1994 [Wepsisch], Saar 2008 [Võru]). 
Die vorgeschlagene Gliederung der Strukturtypen der Namen ist detailliert und knüpft an die fennistische Tradition an. Stellenweise könnte die Analyse durch Verweise auf andere finnisch-ugrische Sprachen neben dem Finnischen Tiefe gewinnen. Während z. B. im Finnischen Reduktionen innerhalb der Namen recht selten vorkommen, sind sie im Karelischen und Wepsischen sehr verbreitet und für die letztgenannte Sprache auch recht gut beschrieben (Mullonen 1994). Ebenso begegnen der lediglich aus einem Bestimmungsglied im Genitiv bestehende Namenstyp, der im südsaamischen Gebiet verbreitet ist, oder der Wechsel von nominativischem und genitivischem Bestimmungsglied äußerst häufig im Estnischen, für das die in den Ortsnamen vorkommenden syntaktischen Typen auch gut beschrieben sind (u. a. Valdek Pall, Maarja Kallasmaa). Ein entsprechender $\mathrm{Na}$ menstyp hat sich auch in den mordwinischen Sprachen z.B. in den Siedlungsnamen verbreitet.

Wie bereits erwähnt, vermittelt die etymologische Analyse der Namensteile weniger den Eindruck einer Primärforschung als der Rest der Untersuchung. Die Verfasserin musste sich hier stärker auf die verfügbaren Quellen und auf ihren im Bereich der Etymologie äußerst kompetenten Betreuer stützen. Ob- wohl dieser Teil nicht wie eine völlig selbstständige Untersuchung erscheint, wird der unterschiedliche etymologische Hintergrund des in den verschiedenen Sprachen begegnenden Wortschatzes (südsaamisch vs. die anderen untersuchten Gebiete) glaubhaft belegt.

Die Schlussfolgerungen der Verfasserin sind durchweg vorsichtig und gut begründet. Sie schreibt im Wesentlichen nur das nieder, was mit Sicherheit festgestellt werden kann. Das ist in vielerlei Hinsicht ein guter Ausgangspunkt. Stellenweise würde das Material jedoch auch kühnere Interpretationen ermöglichen. U.a. scheint das südsaamische Material meiner Ansicht nach auf eine Situation hinzudeuten, in der sich das Saamische in dem Gebiet später verbreitet hat als die skandinavischen Sprachen. Die saamischsprachige Bevölkerung hätte dann die wenig genutzten Hochlandregionen übernommen und die Kontakte zu den Skandinaviern wären an der Waldgrenze intensiver gewesen.

$\mathrm{Zu}$ einem gewissen Grad überraschend erscheint mir die Schlussfolgerung der Verfasserin, die Sprachkontakte im Gebiet von Suonikylä und Dálvadaš seien geringfügig gewesen. Man weiß jedoch, dass die Bewohner dieser Gebiete wichtige Kontakte in vielen Richtungen hatten. Die Frage, 
weshalb ihr Material diese Kontakte nicht widerspiegelt, wird von der Verfasserin nicht hinreichend problematisiert.

\section{Zum Schluss}

Taarna Valtonen hat das erste Gesamtbild der saamischen Ortsnamen vorgelegt und damit einen wichtigen Beitrag zur humanistischen Forschung und zur Kenntnis der saamischen Sprachen geleistet. Ihre Untersuchung bietet auf vielen Ebenen neue, grundlegende Informationen, die von dauerhaftem Wert sind und auf die verwiesen werden wird, solange die saamischen Namen erforscht werden. Darüber hinaus enthält sie zahlreiche interessante Interpretationen und Entdeckungen, die auf die Notwendigkeit zusätzlicher Untersuchungen hinweisen.

Janne Saarikivi

\section{Literatur}

Berezovic, E. L. 2012 = Березович, Е. Л.: Русская топонимия в в этнолингвистическом аспекте. Уральский государственный университет. Екатеринбург.

DMitrieva 2005 = Дмитриева, Т.Н.: Топонимия бассейна реки Казым. Издательство Уральского университета. Екатеринбург.

Kiviniemi, Eero 1990: Perustietoa paikannimistä. Suomalaisen Kirjallisuuden Seura. Helsinki.

Leino, Antti 2007: On toponymic constructions. As an alternative to naming patterns in describing Finnish lake names. Studia Fennica Linguistica 13. Finnish Literature Society. Helsinki.

Mullonen 1994 = Муллонен, И.И.: Очерки вепсской топонимии. ИЯЛИ КарНЦ РАН. Наука. Санкт Петербург.

SAAR, EvAR 2008: Võrumaa kohanimede analüüs enamlevinud nimeosade põhjal ja traditsionioonilise kogukonna nimesüsteem. Dissertationes philologiae estonicae Universitatis Tartuensis 22. Tartu Ülikool. Tartu.

ViInanen, Voitto VAlio 2002-2006: Inarin rajahistoria I-II. Studia Historica Septentrionalia 40 \& 50. PohjoisSuomen historiallinen yhdistys. Rovaniemi. 